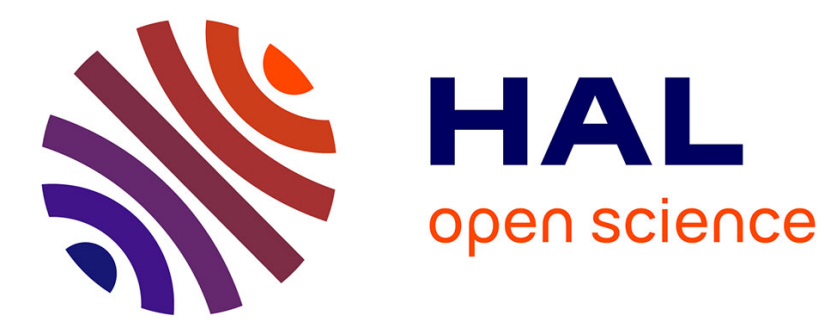

\title{
Histoire et Causalité
}

Jochen Hoock

\section{To cite this version:}

Jochen Hoock. Histoire et Causalité. Revue de Synthèse, 2010, 131 (1), pp.154-160. 10.1007/s11873009-0115-2 . hal-00578387

\section{HAL Id: hal-00578387 \\ https://hal.science/hal-00578387}

Submitted on 20 Mar 2011

HAL is a multi-disciplinary open access archive for the deposit and dissemination of scientific research documents, whether they are published or not. The documents may come from teaching and research institutions in France or abroad, or from public or private research centers.
L'archive ouverte pluridisciplinaire HAL, est destinée au dépôt et à la diffusion de documents scientifiques de niveau recherche, publiés ou non, émanant des établissements d'enseignement et de recherche français ou étrangers, des laboratoires publics ou privés. 


\title{
PREMIÈRES JOURNÉES DE SYNTHÈSE HISTOIRE ET CAUSALITÉ
}

\author{
Wolfenbüttel, Herzog August Bibliothek \\ 11-13 mai 2009, Bibelsaal
}

Jochen HoocK*

$\mathrm{E}^{\mathrm{n}}$ rnst Cassirer relève dans le troisième volume de sa Philosophie des formes symboliques les difficultés que rencontre tout récit strictement causal qui n'est viable que dans la mesure où les données fragmentaires et défectueuses, sur lesquelles il prend appui, sont considérées comme faisant partie d'un tout et perçues sous l'angle de la continuité et de la cohérence ${ }^{1}$. S'agissant de l'historiographie, le problème surgit dès la fin $\mathrm{du} \mathrm{XVII}{ }^{\mathrm{e}}$ siècle et reste un thème constant des débats entre historiens et philosophes. Le dernier a avoir abordé cette question est Paul Ricœur qui a développé, sous le titre La Mémoire, l'histoire, l'oubli², à la fois une phénoménologie de la mémoire, une épistémologie de l'histoire et une herméneutique de la condition historique. Toutefois, il ne se confronte guère à la question d'une épistémologie autonome de l'histoire ${ }^{3}$. Quant aux historiens eux-mêmes, ils ont généralement opté pour une intégration narrative des divers aspects du problème, dont l'œuvre de Fernand Braudel est l'exemple accompli.

Les Journées de synthèse, organisées à Wolfenbüttel, ont abordé ce problème dans une double perspective, à la fois historique et épistémologique, en confrontant les points de vue de différentes disciplines en apparence aussi éloignées que l'histoire des idées politiques et la physique théorique, en incluant l'histoire des sciences et de la philosophie. Renouant avec un débat qui avait opposé en mai 1944 Gaston Bachelard à un

* Jochen Hoock, né en 1939, est professeur émérite d'histoire moderne et contemporaine de l'université de Paris 7, Denis Diderot (2, place Jussieu, 75005 Paris; jochen.hoock@wanadoo.fr). Ses recherches portent sur la théorie de l'histoire. Il a notamment publié, en collaboration avec Pierre Jeannin et Wolfgang Kaiser, Ars Mercatoria. Manuels et traité à l'usage des marchands, 1470-1700 (Paderborn, Schöningh, 1991-2001, 3 vol.).

1. Ernst Cassirer, Philosophie der symbolischen Formen, Dritter Teil: Phänomenologie der Erkenntnis, éd. Volker BAUER, Darmstadt, Wissenschaftliche Buchgesellschaft, 1994, p. 375 sqq.

2. Paul Riceur, La Mémoire, l'histoire, l'oubli, Paris, Le Seuil, 2000.

3. À ce propos, voir Alexandre Escudier, "Épistémologie et ontologie de l'histoire », dans Autour de La Mémoire, l'histoire, l'oubli de Paul Ricœur, numéro spécial du Débat (n 122 , novembre-décembre 2002, p. 12-23, et la réponse de Paul Ricœur, p. 45-51). Concernant les rapports entre théorie de l'histoire et herméneutique philosophique, voir Reinhart KosELLECK, «Théorie de l'histoire et herméneutique. Allocution prononcée à l'occasion du $85^{\mathrm{e}}$ anniversaire de Hans-Georg Gadamer », dans ID., L'Expérience de l'histoire, éd. et trad. Michael WERNER et Alexandre EscuDIER, Paris, Gallimard, 1997, p. 180 sqq. 
groupe d'historiens et de philosophes réuni par Henri Berr ${ }^{4}$, ces journées se donnaient pour but de renouveler ces confrontations régulières sous la forme d'un séminaire ouvert, ayant trait à l'évolution théorique dans le domaine des sciences de l'homme. La volonté d'associer à cette entreprise non seulement plusieurs disciplines, mais encore un nombre aussi large que possible d'institutions universitaires et savantes motivait le choix du lieu de la confrontation et des participants ${ }^{5}$.

La première journée s'est attachée aux controverses des $\mathrm{XVII}^{\mathrm{e}}$ et $\mathrm{XVIII}{ }^{\mathrm{e}}$ siècles qui ont précédé la révolution kantienne et la naissance de l'historisme. Il s'agissait de prendre la mesure à la fois de l'éclatement du champ discursif auquel s'attache la notion d' « histoire » et d'en dégager les implications épistémologiques. Laurie Catteeuw a montré qu'un lien décisif se noue entre la naissance du réalisme politique moderne et les modalités d'observation et de description de la réalité historique. Certaines des transformations qui sont à l'œuvre dans l'élaboration de la rationalité politique moderne font évoluer la conception de la causalité comme cause finale: c'est le cas lors de la reconnaissance d'un impossible accord entre les règles morales et les intérêts politiques, ou encore, lors de l'acceptation, au besoin, de la prévalence de ces derniers. Par ces évolutions qui touchent la conception même de la cause finale, des phénomènes similaires, advenus dans le passé et dans le présent, ne sont plus décrits et analysés de la même manière. Ils sont ainsi perpétuellement "réécrits » (Koselleck). Deux types de relation causale ont joué un rôle moteur au sein de ces évolutions : d'une part, la relation entre causalité et rationalité; d'autre part, la relation entre causalité et nécessité. Le premier aspect a été traité à partir de la réflexion menée par nombre de penseurs et d'historiens de l'époque moderne sur les causes de la grandeur ou de la décadence de telle ou telle cité, de tel ou tel peuple, et en fonction de la place attribuée à la censure dans leur processus de conservation. Le second aspect portait principalement sur la maxime nécessité ne connaît point de loi et sur la réponse, qui peut lui être faite, en terme de « causalité légale » selon l'expression utilisée par Henri Berr lors des débats du Centre international de synthèse sur la notion de causalité. Le rapport entre la causalité et la nécessité constitue le lieu, dans les arts de gouverner, où la question de l'aléatoire est posée. La capacité des gouvernants à prévoir les phénomènes aléatoires, les circonstances improbables à partir desquelles leurs actes prennent sens, devient un enjeu fondamental. Ainsi, le calcul de l'imprévisible, de l'aléatoire en politique appartient pleinement aux arts de gouverner.

Plusieurs interventions ont ramené le discours historique à une multiplicité de textes qui tendent à remplacer l'historiographie annaliste traditionnelle, tel l'essai historique, l'exposé encyclopédique, les collections historiques au sens le plus large du terme incluant l'histoire naturelle et la statistique. L'évolution du discours historique reflète les interrogations soutenues par un scepticisme modéré dans lequel le souci de la preuve, y compris par l'impossible (Balthazar Bekker), a son importance. Jusqu'à quel point

4. Voir «Causalité - Séance du 17 Mai 1944 », dans Les Mots de l'histoire. Le vocabulaire historique du Centre international de synthèse, éd. Margherita Platania, Naples, Bibliopolis, 2000, p. 149 sqq.

5. La rencontre avait, outre le soutien accordé par la Maison des sciences de l'Homme de Paris, celui de la bibliothèque Augusta, des universités de Bielefeld et de Tübingen, et de la Mission historique française en Allemagne. 
ces interrogations pouvaient intégrer des considérations probabilistes évoluant entre une tradition rhétorique et un calcul du probable, c'est ce que s'est attaché à montrer Jean-Pierre Schandeler dans son analyse des rapports de l'Académie des inscriptions et belles-lettres, dont les reconstitutions généalogiques croisaient les approches philologiques et statistiques.

Se tournant vers les enjeux épistémologiques des controverses scientifiques de la fin du $\mathrm{XVII}^{\mathrm{e}}$ siècle, la communication de Jean-Pierre Cléro a décrit et analysé la situation paradoxale qui apparaît avec les interventions de Pascal et Jacques Bernoulli, sans doute aussi avec Locke, et qui se prolonge au XVIII ${ }^{\mathrm{e}}$ siècle, chez Berkeley, Euler, Clairaut, Hume, d'Alembert, puis Laplace. Ce paradoxe consiste à « mettre en pièces » la notion de causalité, en s'interrogeant sur leur articulation les unes avec les autres, sans récuser la puissance de construction que cette notion est susceptible d'opérer. Mais, ce n'est là qu'une partie du paradoxe, sa partie épistémologique car, selon Jean-Pierre Cléro, il en est une autre, plus métaphysique.

Même aux yeux de ceux qui sont le plus enclins à adopter un point de vue sceptique à l'égard des causes, il est bien évident que le déterminisme et la nécessité des processus règnent sans partage. Cette affirmation peut paraître étrange car autant la cause est critiquée par une conscience du point de vue que l'on adopte sur les phénomènes, autant le déterminisme est posé en soi, sans connaissance ni point de vue. Ce dogmatisme, en toile de fond du scepticisme le plus aigu à l'égard des causes, inquiète par son apparente inconséquence qui suppose que les frêles esquifs de causes (que nous inventons) allaient à la rencontre d'une nécessité qu'il faut découvrir plutôt qu'inventer. À y regarder de plus près, il semble que cette dialectique de l'invention et de la découverte dans le travail intellectuel se soit particulièrement jouée dans les Principia mathematica, qui ont pu donner l'illusion que l'invention des causes, réglées par des lois, coïncidait avec la détermination des choses. La loi d'attraction, le principe d'inertie, la puissance de l'analyse nouvelle se combinaient pour donner l'impression que l'on parlait des choses en complicité avec leur Auteur divin. L'illusion était d'autant plus forte, qu'elle concernait des objets situés si loin de nous qu'il n'était pas question de les manipuler, mais seulement de les observer, nous reléguant à une sorte de passivité fondamentale qui sied à l'affirmation et au renforcement de la conviction du déterminisme. Celui qui se vantait de « ne pas faire d'hypothèses » pouvait avoir le sentiment ou donner l'impression que l'attraction et l'inertie étaient celles des choses. Or, cette superbe conjonction de l'esprit humain, des choses et de l'intention divine n'a d'abord jamais été parfaite et Newton, qui l'établit, ne pouvait pas ignorer les multiples imperfections de son propre système. Elle n'a fait ensuite que se dégrader au cours du XVIII ${ }^{\mathrm{e}}$ siècle, laissant les savants dans l'alternative soit de renoncer au déterminisme, ce qu'ils n'osent faire au moins dans leurs affirmations les plus exotériques, soit d'inventer, grâce à la puissance de l'instrument sans cesse renouvelée du calcul, un jeu de corrections des causes les unes par les autres. C'est cette dernière voie qu'ils choisiront, pour la plupart d'entre eux, corrigeant la loi d'attraction. Le ciel, la mécanique céleste, qui semblaient la meilleure caution du newtonisme, vont se montrer ingrats avec les Principia. Les « restes » que produit l'explication newtonienne vont constituer l'essentiel du souci des astronomes: leur travail tente de réduire l'exaction que les phénomènes observés paraissent représenter à l'égard de la loi universelle à laquelle ils 
devraient se plier. Mais pourquoi ces auteurs, qui sentent bien les efforts qu'il leur faut faire pour se hisser à l'unisson d'un prétendu déterminisme, n'ont-ils pas renoncé à la fière affirmation du déterminisme?

C'est au tournant provoqué par les débats soulevés par les tenants de l'école dite de Göttingen que s'est attachée l'intervention de Jochen Hoock. Phénoménomalité, causalité et temporalité sont, au moment où Gatterer et Schlözer développent leur " plan historique », au centre des controverses que soulèvent les travaux de Johannes Nikolaus Tetens: ces derniers popularisent en Allemagne les thèses de David Hume et Jean-Baptiste-René Robinet, dont les considérations philosophiques sont parues au cours des années 1760. La question de savoir ce qui explique un phénomène et ce qui le porte, sa dimension épistémologique et sa dimension ontologique, entre dans une constellation philosophique qui prend l'allure d'un vaste débat mobilisant l'ensemble du public savant et littéraire. Le projet de l'école de Göttingen paraît dans ce contexte théoriquement démuni en se donnant un modèle analytique qu'elle n'est capable ni de verbaliser de façon adéquate ni de représenter sous une forme symbolique acceptable. L' « objectivisme » de Schlözer se réfère à une « causalité naturelle » que de nombreux autres participants au débat s'efforcent de sauver: soit en recourant à certaines figures wolffiennes, soit en épousant des positions théologiques qui vont de la théologie de la création aux courants spiritualistes de la deuxième moitié du XVIII ${ }^{\mathrm{e}}$ siècle.

Concernant l'historiographie, une première réaction vient de Herder: il élabore un modèle de récit historique qui emprunte sa cohérence au texte littéraire, en proposant un schéma transcendantal permettant de réunir les éléments disparates des événements observés. Tout le cercle d'intellectuels, dont Jean Quillien a retracé les débats pendant la période révolutionnaire, contribua jusqu'à la publication de l'essai de Guillaume de Humboldt sur la Tâche de l'historien (1821) à redéfinir les rapports entre poétique et histoire, dont Diderot avait relevé l'importance stratégique dans son Éloge de Richardson dès 1761. La pression des événements, les bouleversements de la fin du siècle font par ailleurs que l'histoire apparaît plus que jamais comme une scienceguide, bien qu'elle n'ait - selon les mots de Kant - pas encore trouvé son Kepler ou son Newton.

Le «fardeau de l'histoire», tel que l'entendait Kant, est à la fin du XVIII siècle un enjeu qui dépasse le débat académique. Il est devenu inséparable de l'extension spatiale du champ d'expérience qui transforme dans le même temps l'expérience proche, comme le montrent les réflexions de Georg Forster dans ses Observations lors d'un voyage au Bas-Rhin. Plus généralement cette mutation reflète l'inexpérience du présent avec laquelle s'impose la mise à plat des différents niveaux d'historicité qui caractérise l'historisme. La réflexion méthodologique qu'elle inspire se retourne au début du XIX ${ }^{\mathrm{e}}$ siècle contre l'ambition de la statistique (Lueder vs Schlözer) avant de laisser la place à l'éclatement disciplinaire des sciences humaines qui vont occuper, à côté de l'histoire de l'économie politique et de la sociologie qui cherche encore son nom, le champ de ce qui relève désormais en termes scientifiques de l'explication et de la compréhension.

La deuxième journée s'est tournée vers les rapports entre l'histoire et les sciences dites exactes : un questionnement auquel invitait la relecture des controverses qui ont fait surgir, à la fin $\mathrm{du} \mathrm{XVIII}^{\mathrm{e}}$ siècle et au début du XIX ${ }^{\mathrm{e}}$ siècle, la différenciation entre 
les paradigmes explicatifs et interprétatifs. Comment et à quel degré ces rapports se sont-ils modifiés depuis les grandes controverses qui ont opposé les historiens entre eux lors du Methodenstreit du début du $\mathrm{xx}^{\mathrm{e}}$ siècle et de l'irruption de l'analyse causale avec les travaux de François Simiand? Revenant sur les travaux d'Erwin Panofski et de Gaston Bachelard, Vincent Bontems à posé l'hypothèse suivante: si l'élaboration d'une épistémologie autonome des sciences historiques suppose la distinction entre le temps historique et le temps physique, la notion de "causalité » en histoire peut être précisée à partir de la comparaison des méthodes entre sciences de la nature et sciences historiques. Dans une telle perspective il est essentiel d'examiner l'articulation entre le temps chronologique, objectif, homogène et orienté, qui sépare et ordonne les événements aussi bien en physique qu'en histoire, et un temps phénoménologique, qui rend compte de ce qui est vécu par les agents dans leur « présent » situé par rapport au passé et au futur. L'hypothèse d'une « causalité historique » entre des événements ne consiste pas à supposer un déterminisme physique continu entre eux: un événement peut être tenu pour la cause historique d'un autre si la façon de vivre le présent des agents prend sens en fonction de la manière dont ils se rapportent à cet autre «référentiel ». Cette reformulation du problème doit beaucoup à l'intégration des implications conceptuelles de la théorie de la relativité restreinte élaborée par Albert Einstein en 1905. La relativité restreinte a constitué une révolution scientifique qui a modifié les relations entre causalité et simultanéité physiques. Certains historiens en ont tiré les leçons en modifiant, par analogie, leur propre conception de la causalité et de la contemporanéité historiques. La notion de causalité historique s'éclaire, en effet, sous un jour nouveau quand, au lieu d'identifier la contemporanéité entre des référentiels historiques à leur simultanéité chronologique, l'on précise la résonance qui existe entre eux, qu'ils soient chronologiquement simultanés ou non. C'est dans cette perspective que la communication se proposait d'interpréter l'influence de la conception relativiste du temps sur l'historien de l'art Erwin Panofsky, qui élabore la notion de « contemporanéité relative », et sur l'historien des sciences Gaston Bachelard, chez qui elle induit la relativisation de la notion de «durée » ainsi qu'une conception discontinue de la temporalité. C'est donc un nouveau concept de causalité historique, fondé sur la consolidation de rythmes, qui émerge de ces travaux.

C'est à la même transformation des rapports de causalité et de l'évaluation de l'incertitude que s'est attaché le débat qui a suivi la communication d'Éric Brian sur la probabilité dans l'écriture chez Condorcet. Le géomètre philosophe, en effet, est connu pour deux contributions à des domaines apparemment fort éloignés : d'une part, la théorie et la philosophie du calcul des probabilités (c'est son œuvre mathématique d'extension du calcul intégral au calcul analytique des probabilités, contemporaine de celle de Laplace); d'autre part, la philosophie de l'histoire et une célèbre tentative de mise en récit des progrès de l'esprit humain (voir l'édition critique des manuscrits relatifs au Tableau historique parue à Paris, aux Éditions de l'Institut national des études démographiques, en 2004). Ces deux pans du corpus condorcétien partaient en leur temps des débats analysés au cours des exposés précédents et leur répondaient. Leur étude attentive montre qu'ils étaient profondément cohérents l'un avec l'autre, chose perdue de vue au siècle suivant du fait de l'essor du positivisme, de l'oubli 
collectif des questions vives du siècle des Lumières et de la divergence des « deux cultures », littéraire et mathématique avec l'essor en Europe de la spécialisation dans l'enseignement de haut niveau. Le succès simpliste de l'Esquisse au XIX ${ }^{\mathrm{e}}$ siècle a donc occulté un chantier que Condorcet aurait pu désigner comme la "métaphysique des sciences de l'homme » où le raisonnement probabiliste, l'analyse des causes et des effets de l'écriture de la science sont en jeu dans un même mouvement. Dans cette perspective, il est possible de réévaluer des auteurs ultérieurs connus mais dont l'épistémologie probabiliste, pourtant attestée, est sous-estimée par les traditions de leurs commentaires, tels John M. Keynes ou Maurice Halbwachs. Il importe sans doute d'examiner à nouveaux frais ces questions en prenant acte des mutations du raisonnement historiographique et du raisonnement stochastique ${ }^{6}$ au $\mathrm{Xx}^{\mathrm{e}}$ siècle et aujourd'hui. Cet exposé a été complété par un exposé de Michael Heidelberger sur les références aux controverses philosophiques contemporaines de la contribution de Gaston Bachelard au débat sur la causalité telles qu'on peut les relever dans son intervention au Centre de synthèse en 1944.

Une référence constante du dialogue engagé lors de la réunion à Wolfenbüttel restait le débat entre historiens tel qu'il avait été noué au début du $\mathrm{xx}^{\mathrm{e}}$ siècle. Interrompu par la guerre, ce débat se poursuivit lors des années 1920 et 1930 dans une sorte de consensus mou autour de l'irruption de l'histoire économique et sociale où Simiand devint une référence constante sans être réellement suivi ou discuté. Le problème de la causalité en histoire est repris par Lucien Febvre en recourant au concept de " crise » et à l'analyse des constellations synchroniques tout en reconnaissant la contribution de l'élève de Durkheim à l'étude des conjonctures et à l'histoire des prix. Mais là où Simiand privilégiait l'abstraction comme mode d'analyse historique, Febvre prône la qualification du phénomène unique dans son époque, dans un «Zusammenhang » dont le critère serait l'anachronisme.

Tout à fait différente était la position de Marc Bloch dont la démarche heuristique semblait partagée par les participants à la réunion de Wolfenbüttel. Là où Febvre parlait d'une « histoire - science des changements », Bloch raisonnait en effet dans les catégories $d u$ changement ${ }^{7}$. « L'histoire - note-t-il vers 1942 dans l'Étrange défaite - est science du changement. » Elle sait qu'aucun événement ne ressemble à un autre. Là où l'historien reconnaît dans l'évolution humaine quelques éléments stables et permanents, il constate au même moment leur variabilité presque infinie. Ce sont en fait les mêmes critères d'intelligibilité, les mêmes références théoriques qui donnent en dernière analyse la mesure de ce que nous reconnaissons comme expression de la pratique humaine passée et son avenir possible.

Si le but de ces journées était dans un premier temps d'établir une sorte d'état des lieux et de s'interroger sur l'autonomie des disciplines dites historiques, sa visée à terme était de jeter les bases des rencontres régulières telles qu'elles se sont tenues au

6. La décade Complexity, Mathematics and Socio-Economic Problems organisée par le ZiF (Center for Interdisciplinary Research), à l'université de Bielefeld, du $1^{\text {er }}$ au 11 septembre 2009, portait sur ce dernier point.

7. Voir Lucien Febvre, « Souvenirs d'une grande histoire: Marc Bloch et Strasbourg », dans ID., Combats pour l'histoire, Paris, Armand Colin, 1952; Marc BLoch, Apologie pour l'histoire ou Métier d'historien, Paris, Armand Colin, 1997, p. 52. 
Centre international de synthèse sous l'égide d'Henri Berr. Le dialogue entre historiens, économistes, physiciens, philosophes et spécialistes de l'histoire des sciences établit à Wolfenbüttel n'est donc qu'un début ${ }^{8}$. Il devrait trouver son moyen d'expression dans les publications régulières de la Revue de synthèse. Parmi les thèmes retenus ont émergé ceux de l'analogie, de l'incertitude et de l'action, ainsi que les questions s'attachant à l'épistémologie historique.

\section{LES PARTICIPANTS}

Volker BAUER (responsable du programme scientifique de la Bibliotheca Augusta, Wolfenbüttel) Philippe BlanchaRd (professeur à l'université de Bielefeld) Hans-Erich BÖDEKER (chercheur à l'Institut Max Planck für Wissenschaftsforschung, Berlin) Vincent BonTEMs (chercheur au Commissariat à l'énergie atomique, Orsay) Éric BRIAN (directeur d'études à l'École des hautes études en sciences sociales, Paris)

Neithard Bulst (professeur émerite de l'université de Bielefeld)

Laurie CATTEEuw (attachée temporaire d'enseignement et de recherche à l'École des hautes études en sciences sociales, Paris)

Jean-Pierre Cléro (professeur à l'université de Haute-Normandie, Rouen)

Guillaume GARNER (chercheur à l'Institut français d'histoire en Allemagne, Francfort)

Michael HeIDELBERgER (professeur à l'université de Tübingen)

Jochen Нооск (professeur émérite de l'université de Paris 7)

Jean-Pierre SCHANDELER (chercheur au Centre national de la recherche scientifique, Montpellier)

8. Notons que la réunion a éveillé la curiosité de la grande presse allemande comme en témoigne l'article de Thomas ThiEL, Die Formeln der Geschichte, paru dans la Frankfurter Allgemeine Zeitung, le 20 mai 2009. 